\title{
Nostalgische SChNitTmuster im JazZ VON CassandRa Wilson, Diana Krall und NORAH JONES
}

\author{
Jürgen Arndt
}

\begin{abstract}
I.
1992 kommentierte Kontrabassist Charlie Haden die CD Haunted Heart seines Quartet West:

»I have conceived this recording as if it were a film telling a story. A story evoking feelings of nostalgia, remembering beautiful moments and how precious they are; a story that evokes the importance of every human being on this earth, and that the history of the universe from the beginning of time is in each of us« (Haden 1992).

Bemerkenswert ist nicht allein die Ausrichtung der Albumkonzeption am Medium Film, sondern auch die damit einhergehende nostalgische Rückbesinnung. Haden präsentierte musikalische Erinnerungen an die 1940er und 1950er Jahre, insbesondere den Hollywoodfilmen dieser Zeit folgend. Zu diesem Zweck wählte er vor allem entsprechende Stücke der genannten Jahrzehnte aus, die er dann mit seinem Quartett interpretierte. Darüber hinaus griff er auch auf Aufnahmen dieser Zeit zurück, deren vollständige Fassungen er in den Ablauf integrierte: von Max Steiners Fanfare für die Filme der Warner Brothers bis zu Interpretationen von Jo Stafford und Billie Holiday. Das auf Haunted Heart vorgestellte Konzept verfolgte Haden mit seinen nachfolgenden Alben weiter. Besonders erfolgreich war dabei die 1994 erschienene CD Always Say Goodbye.

Mit Charlie Haden ist beispielhaft ein Musiker mit einem deutlichen Hang zur musikalischen Nostalgie genannt, einem herausragenden jüngeren Trend im Jazz. Weitere prominente Namen und Projekte lassen sich anfügen. Cassandra Wilson, Diana Krall und Norah Jones, die zu den erfolgreichsten Jazzstars der letzten Jahre gehören, zählen ebenfalls dazu.
\end{abstract}


Nostalgische Sujets wie das Heimweh oder die Sehnsucht nach vergangenen Zeiten lassen sich weit zurückliegend in den verschiedensten Genres finden. Zumeist wurden diese Sujets jedoch in musikalischer Hinsicht durch jeweils aktuelle oder gar modische Stile mit einem sentimentalen Unterton unterstützt, ohne dass deshalb schon der verlassene Ort oder die zurückliegende Zeit erklungen wären. Dagegen lässt sich von musikalischer Nostalgie sprechen, wenn die klanglichen Ausprägungen selbst zusätzlich sehnsuchtsvoll auf vergangene Musikstile und damit einhergehende Assoziationen hin ausgerichtet sind.

\section{Cassandra Wilson}

Zwar wurde Cassandra Wilson in den 1980er Jahren als Mitglied des avantgardistisch ausgerichteten M-Base-Zirkels um Steve Coleman bekannt und zielte mit ihren eigenen Projekten eher auf klangliche Modernisierungen traditioneller Elemente. Mit ihrem Wechsel zu dem renommierten Label Blue Note schlug sie seit 1993 jedoch eine andere Richtung ein und begab sich auf ihren ganz eigenen nostalgischen Pfad. Hilfreich erwies sich dabei die Unterstützung ihres Produzenten Craig Street. Wilson hatte Street zuvor im Plattengeschäft ein unbeschriebenes Blatt - bei einem von ihm organisierten Tribute-Projekt zur Musik von Jimi Hendrix kennen und schätzen gelernt und ihrer neuen Plattenfirma vorgeschlagen.

Ganz ähnlich wie Haden, wenn auch nicht von vornherein nostalgisch ausgerichtet, assoziiert Street seine Arbeit mit dem Film:

»Ich nähere mich den Produktionen an, als wären es Filme oder ein großartiges Essen, das es vorzubereiten gilt. Wenn es beim Mix um die Kolorierung und die richtige Balance geht, frage ich mich, wie bestimmte Fotografen oder Architekten dieses Problem lösen würden. Wie würde die Produktion bei [Robert] Rauschenberg funktionieren? Bei [Wim] Wenders oder [Federico] Fellini?« (Craig Street, zit. n. Kampmann 2000: 56).

Die Zusammenarbeit zwischen Cassandra Wilson und Craig Street brachte entsprechend Montagen verschiedener differenziert gewählter und arrangierter Klangbilder hervor. Das gilt sowohl für das 1993 veröffentlichte Album Blue Light 'Til Dawn wie auch für das zwei Jahre später erschienene Nachfolgealbum New Moon Daughter. Mit dem klanglich abwechslungsreichen Ansatz korrespondiert das vielfältige Songrepertoire von CountryBlues-Klassikern, Evergreens des Great American Songbook, jüngeren Popsongs und Eigenkompositionen Wilsons. 
Für den Zusammenhalt sorgt dabei die durchgehend musikalisch-nostalgische, ländliche Atmosphäre heraufbeschwörende Ausrichtung. Sie zeigt sich zunächst in der pointierten Herausstellung fast ausschließlich akustischer Instrumente von verschiedenen, gerade im Süden der USA gebräuchlichen Dobros (Resonatorgitarren mit metallenem Korpus), diversen Perkussionsinstrumenten bis hin zu Violine und Kontrabass. Es entstehen jeweils eher zurückhaltende, sparsame Klangbilder, in deren Mix die eingesetzten Instrumente eine je dezidiert eigene Betonung erhalten. Nicht minder unmittelbar wird die Stimme von Cassandra Wilson herausgestellt. Ihre verschiedenen Facetten und die daraus resultierenden Nuancen sind jederzeit besonders präsent. Auf höchstem aufnahmetechnischen Niveau entsteht hier ein scheinbar unverstellt intimer, ländlich anmutender Sound.

Dieser Klang wandelt sich im Verlauf des Albums von Stück zu Stück. Das Kontinuum wird aufgebrochen; der Sound wird als stilisierter und nicht etwa als vermeintlich authentischer vorgeführt. Die suggerierte ländliche Idylle wird als vergangene nostalgisch erfahrbar.

Mit ihrer Interpretation des Songs "You Don't Know What Love Is« von Gene DePaul und Don Raye aus dem Jahr 1941 gibt Wilson - begleitet von Gitarrist Brandon Ross und Geiger Charles Burnham - einleitend das Motto des Albums vor: "You don't know what love is, until you know the meaning of the blues«. Die Rückbesinnung auf den Blues, insbesondere auf den traditionellen Mississippi Delta Blues stellt einen wesentlichen Bezugspunkt von Wilsons Album Blue Light 'Til Dawn dar. Sogar auf zwei Titel von Robert Johnson greift Wilson zurück. Sie erinnert damit an den herausragenden musikalischen Beitrag Johnsons einerseits wie auch an den Kultstatus, den Johnson als berühmtester Vertreter des Mississippi Delta Blues mit seinen Aufnahmen aus den 1930er Jahren posthum erlangte.

Die klangliche Distanz zwischen der Einspielung Johnsons von 1936 und derjenigen von Wilson aus dem Jahr 1993 könnte nicht größer sein. Und dennoch beschwört Wilson gerade durch die klanglichen Eigenheiten ihrer Version die vergangene Musik eher herauf, als wenn sie diese klanglich möglichst getreu nachgeahmt hätte. Dadurch wäre allenfalls eine überflüssige Kopie entstanden.

Das schon angesprochene, aufgebrochene Klangbild des gesamten Albums kristallisiert sich in Wilsons Interpretation von Robert Johnsons "Come On In My Kitchen« besonders heraus. Das Arrangement von Brandon Ross führt die eingesetzten Instrumente nicht homogen zueinander, sondern setzt diese einzeln akzentuiert ein. Das zeigt sich in dem kurzen, fragil intonierten Gitarrenrhythmus, im ostinat eingesetzten Kontrabass-Glissando in betont tiefer Lage und besonders auch im gesplitteten Einsatz des Schlag- 
zeugs, dessen Elemente nicht simultan, sondern sukzessiv erklingen. BassDrum, Rimshot auf der Snare und das Hi-Hat bilden - nacheinander eingesetzt - das grundlegende Begleitpattern. Auf dieser klanglich offenen Grundlage bringt Wilson ihr tiefes, samtiges Timbre in den verschiedensten intimen Schattierungen zur Geltung. Durch ihren eher zurückhaltenden Gesang bekommt das aufgebrochene Klangbild des Songs den Charakter des Nachhalls. Eine Einschätzung, die durch das letzte Drittel der Version von »Come On In My Kitchen« noch zusätzlich an Plausibilität gewinnt. Durch die Ausdehnung auf etwa zwei Minuten lässt sich diese Passage nicht mehr nur als Schluss auffassen, sondern bekommt einen eigenen, wichtigen Stellenwert. Der Echo-Effekt wird hier zugespitzt. Wilson beginnt im Anschluss an die dritte Strophe mit dem Text klanglich-spielerisch zu improvisieren, stellt sich dadurch aber nicht noch mehr in den Vordergrund, sondern zieht sich bald zurück, um nur noch gelegentlich einsetzend vereinzelte Fragmente anzudeuten. Wenn sie dann die Titelzeile des Blues aufgreift, singt sie diese gleich mehrfach unmittelbar hintereinander und wiederholt sie immer leiser werdend.

Über die Anlehnung an den Country Blues hinaus ist eine zweite musikalische Bezugnahme für das Album Blue Light 'Til Dawn wichtig und wird nicht minder stilisiert präsentiert. Bei mehreren afrikanisch anmutenden Stücken wird der Gesang ausschließlich durch übereinander geschichtete Perkussionspattern begleitet. Dem eher melodiös-schwebenden Gesang steht hier ein belebtes Rhythmusgeflecht gegenüber. Ein Klangkonzept, das Wilson auf ihrem 1992 veröffentlichten Album After The Beginning Again, das Blue Light 'Til Dawn unmittelbar voranging, bereits bei einem Titel erprobt hatte und nun auf mehrere Stücke ausweitete. Sie griff sogar denselben Titel, ihre Eigenkomposition »Redbone«, wieder auf.

Mit dem Nachhall auf den Country Blues und afrikanisch stilisierte Perkussionsgeflechte inszeniert Cassandra Wilson eine bodenständige, ursprüngliche Sinnlichkeit, wie sie auch in ihren beiden jüngeren CDs in je eigenen Ausprägungen erneut zum Tragen kommt. Nach dem Album Rendezvous (1997) mit Pianist Jacky Terrasson und Traveling Miles (1999), einer Hommage an Miles Davis, setzte Wilson 2002 mit Belly Of The Sun den auf Blue Light 'Til Dawn eingeschlagenen Weg fort. Die Konzeption von Belly Of The Sun ist folglich ähnlich. Wilson agiert nun auch selbst als Produzentin. Bemerkenswert ist die Entstehungsweise des Albums, mit der Wilson den mit Craig Street entwickelten Ansatz weiter zuspitzt: Sie kehrte nicht nur musikalisch ins Mississippi Delta zurück, sondern nahm das Album nun sogar vor Ort in einem alten Bahnhof in Clarksdale auf. In den zehn Aufnahmetagen im August 2001 sollte nicht nur die sommerliche Hitze für eine 
prägende Atmosphäre sorgen. Durch den Austausch mit im Mississippi Delta ansässigen Musikern wie Pianist Boogaloo Ames und Gitarrist Jesse Robinson entstand insbesondere eine noch größere musikalische Vielfalt. Michael Simanga berichtet im Begleittext zur CD:

"What began as a blues-based record became an experience in creative discovery. The sessions in Mississippi explored blues, jazz, African, Brazilian, pop and R\&B. It ventured into social issues and spiritual life, love and lust, the contemplative and common« (Simanga 2002).

Simangas Verallgemeinerungen, ja Überhöhungen der Atmosphäre dienen der Inszenierung des Albumkonzepts ebenso wie die graphische Gestaltung des Booklets. Mit vergilbt gefärbten Seiten und den ins Braungelbliche getönten Fotos wird die nostalgische Ausrichtung auch visuell unterstrichen.

Mit ihrem jüngsten Album Glamoured (2003) setzt Wilson dann einen etwas moderneren Akzent. Zwar sind erneut einige Aufnahmen in den Südstaaten entstanden (dieses Mal in Wilsons Geburtsort Jackson) und auch die grundlegend nostalgische Orientierung ebenso wie das damit einhergehende akustische Klangbild bleiben erhalten. Dennoch bekommt die CD durch die Mitwirkung des HipHop-Produzenten und Gitarristen Fabrizio Sotti einen aktuelleren Anstrich, gerade durch den gelegentlich eher rhythmisch und weniger melodisch geprägten Gesang.

\section{Diana Krall}

»There may be troubles ahead. / But while there's moonlight and music and love and romance, / Let's face the music and dance. « Mit diesen Versen und der dazugehörigen aufmunternden Musik hat Irving Berlin im Jahr 1936 gewissermaßen das Credo sentimentaler Popmusik vorgestellt. Um »moonlight and music and love and romance « ranken sich alle Schlager, die eine heile Liebeswelt jenseits des Alltags beschwören. Einst für den Film Follow The Fleet mit Fred Astaire und Ginger Rogers komponiert, eroberte sich der Song wie viele andere Songs von Berlin einen prominenten Platz im so genannten Great American Songbook. ${ }^{1}$ Diana Krall eröffnet mit »Let's Face The Music And Dance ihr insgesamt fünftes Album, When I Look In Your Eyes, aus dem Jahr 1999.

1 "Let's Face The Music And Dance wird im Film von Fred Astaire gesungen. Darüber hinaus nahm Astaire den Song parallel zur Entstehung des Films am 30. Januar 1936 in Los Angeles noch für eine Schallplattenveröffentlichung auf. 
Auf den beiden vorangegangenen CDs hatte Krall eine eher intime Triobesetzung gewählt und sich unter Verzicht auf einen Schlagzeuger von Gitarre und Kontrabass begleiten lassen, durchaus in Anlehnung an das berühmte Trio von Nat King Cole aus den 1940er Jahren. Cole, selbst romantischer Sänger und brillanter Pianist, bildet sicher einen entscheidenden Bezugspunkt für die Entwicklung Kralls. Nicht zufällig handelt es sich bei ihrem dritten Album All For You um eine Hommage an Cole und sein damaliges Trio.

Auf When I Look In Your Eyes zieht Krall bei den meisten Titeln einen Schlagzeuger heran, bei etwa der Hälfte der Stücke tritt ein Orchester hinzu. Die Arrangements gehen auf Johnny Mandel zurück. Mandel, ein Freund von Kralls Produzent Tommy LiPuma, hat sich seit den 1950er Jahren mit vielen populären Projekten einen herausragenden Namen als Komponist und vor allem als Arrangeur gemacht. ${ }^{2}$

Neben Berlins Evergreen entsteht also durch die Mitwirkung Mandels ein zweites retrospektives Moment. Die nuancierten Orchestereinsätze geben dem Comboklang zusätzliche Farbtupfer, ergänzen aufgrund ihres instrumentations- wie produktionstechnisch dezenten Einsatzes den Gesamtklang, prägen ihn jedoch nur äußerst zurückhaltend. Das wird gleich in der Einleitung deutlich, etwa im Einstieg der Harfe als Ergänzung zur Gitarre oder im ersten gehaltenen Streicherakkord. Weder Kralls Klavierspiel noch ihr Gesang schweben auf einem orchestralen Klangteppich.

Ein drittes retrospektives Moment kommt ebenfalls gleich zu Beginn der Einspielung von »Let's Face The Music And Dance« zur Geltung. Krall verkleidet Berlins Song als Bossa Nova und gibt ihm damit eine zusätzliche historische Note. Da die Bossa Nova als Verschmelzung von brasilianischer Samba und nordamerikanischem Song entstanden ist, verliert auch »Let's Face The Music And Dance « nichts von seiner homogenen Anlage. Es verwundert darum auch nicht, dass Kralls Vorgehen, einen Song Berlins als Bossa Nova zu interpretieren, nicht einmal originell ist. Ähnliches hatte Frank Sinatra schon 1967 mit Berlins »Change Partners« auf einem gemeinsam mit Antonio Carlos Jobim eingespielten Album vorgemacht. Damals in den 1960er Jahren war die Bossa Nova jedoch noch modisch gegenwärtig; Sinatra aktualisierte also Berlins Song. Krall erhöht demgegenüber mit der Bossa Nova-Variante das vergangene Potential der Musik. ${ }^{3}$ Erste nostalgische

2 Mandel arbeitete als Arrangeur u. a. für Frank Sinatra, Tony Bennett sowie Barbra Streisand und schrieb zahlreiche Filmmusiken, z.B. 1958 für I Want To Live und 1969 für MASH.

3 Jamie Reids Einschätzung von Kralls Version von »Let's Face The Music And Dance « - »The lightly syncopated rhythms of the Argentine [sic!] bossa nova played under the lyrics of this often-heard song helps to revive the music and 
Züge entstehen schon durch das zurückgenommene Bossa-Nova-Tempo, wodurch der aufmunternde Charakter von »Let's Face The Music And Dance nurmehr gedämpft daherkommt. Das Lied wird gleichsam zum Nachhall seiner selbst. Die Eigenständigkeit von Kralls Interpretation ist jedoch noch viel weitreichender und zeigt sich insbesondere in ihrem Gesang. Teilweise verzögert sie den Einsatz der Melodietöne extrem und gibt ihnen mit einer Mischung aus gehauchter Intimität und lakonischer Distanz einen ganz eigenen Charakter. In ihrem Stimmklang und ihrer Phrasierung spiegelt sich die nostalgische Diskrepanz zwischen sehnsuchtsvoller, letztlich unerfüllter Erinnerung und der eigentlich abgeschlossenen Vergangenheit. Die von Krall suggerierte Intimität bekommt zugleich den Zug des Unnahbaren.

Auch Mandels Arrangement ist in diesem Zusammenhang noch einmal der Erwähnung wert. Für »Let's Face The Music And Dance« hatte er schon einmal - fast vierzig Jahre zuvor - ein Arrangement geschrieben: Für RingA-Ding Ding!, das erste Album auf Sinatras eigenem Label Reprise Records, steuerte Mandel 1960 die Arrangements bei. "Let's Face The Music And Dance « wird hier als optimistisch swingende Big-Band-Nummer präsentiert; der Gegensatz zur Aufnahme mit Krall könnte kaum auffälliger sein.

Dass Diana Krall im Unterschied zur direkten Präsenz Sinatras ihre Version eher als nostalgischen Nachklang auf längst vergangene musikalische Zeiten inszeniert, zeigt auch der gedehnte Schluss. Dieser nimmt mit seiner zweiminütigen Dauer einen außergewöhnlichen Anteil an der insgesamt gut fünfminütigen Einspielung ein. Immer wieder greift sie den Titelvers von neuem singend auf, wiederholt inn, ohne dass deshalb der Song lebendiger würde. Auch durch Wechsel zwischen Gesang und kurzen Klavier- bzw. Orchesterzwischenspielen entsteht keine neue Richtung mehr. Krall zelebriert den Song als nachklingendes Echo, durchaus in dieser Hinsicht verwandt mit Cassandras Wilsons Version von »Come On In My Kitchen«.

Sowohl die Wahl des Songs von Irving Berlin als auch der Rückgriff auf die Bossa Nova, aber ebenfalls der betonte Hauch in ihrer Stimme zeigen das Klischeehafte der Interpretation von "Let's Face The Music And Dance . Dass es sich dabei keineswegs um ein kreatives Manko handelt, sondern gerade hierin der intendierte künstlerische Ansatz liegt, bestätigt Krall in einem anderen, verwandten Zusammenhang.

Zwei Jahre nach dem Album When I Look In Your Eyes präsentierte Krall 2001 ihre nächste CD: The Look Of Love. Bestehend ausschließlich aus Balladen und Bossa Novas, spitzt Krall den stilistisch und klanglich noch offeneren Charakter des Vorgängeralbums hier entschieden zu. Erneut kommen

lends the lyrics a fresh, contemporary feeling« (Reid 2002: 158) - ist entschieden zu widersprechen. 
Orchesterarrangements zum Tragen, dieses Mal geschrieben von Claus Ogerman. Nicht minder arriviert als Mandel, war Ogerman bezeichnenderweise der Arrangeur des schon erwähnten Albums von Sinatra und Jobim aus dem Jahr 1967. Den Titelsong »The Look Of Love«, eine Komposition von Burt Bacharach von 1967, interpretierte Krall nicht nur musikalisch, sondern darüber hinaus auch in einem dazugehörigen Videoclip. Das Klischeehafte, ja geradezu Plakative des Krallschen Ansatzes wird selbst zum Thema des Clips. Krall erscheint überlebensgroß auf Plakaten an einer Häuserzeile. Die Plakatbahnen werden von zwei jungen Arbeitern angeklebt; Passanten bleiben stehen, gehen weiter. Die >Plakate - dienen als Projektionsfläche für die filmisch bewegte, intim inszenierte Performance von Diana Krall. Damit steht die musikalisch-nostalgische Interpretation von »Let's Face The Music And Dance als prägnantes Beispiel nicht nur für das Album When I Look In Your Eyes, sondern auch für das Nachfolgealbum The Look Of Love.

Bei der stilisierten Künstlichkeit ist Diana Krall nicht stehen geblieben. Mit ihrem Album The Girl In The Other Room von 2004 nimmt sie wesentliche Neuerungen an ihrer nostalgischen Ausrichtung vor. Nun sind es nicht mehr die alten Evergreens, sondern Songs jüngerer prominenter Singer/ Songwriter, auf die sie zurückgreift: Songs von Joni Mitchell, Tom Waits und Elvis Costello. Noch bemerkenswerter sind die Eigenkompositionen, die Krall erstmals in ihr Repertoire aufnimmt. Die Texte schrieb sie zusammen mit Costello, für die Musik zeichnet sie allein verantwortlich.

Bereits in der persönlich anmutenden Auswahl der Songs von Mitchell, Waits und Costello begibt sich Krall ein wenig abseits des Mainstream. Erst recht zeigen ihre eigenen Songs eine persönliche, ja private Seite, die sie bislang ausgeblendet hatte. Die Titel sprechen eine deutliche Sprache: »The Girl In The Other Room« als das Titelstück des Albums, »|'ve Changed My Address« oder »Abandoned Masquerade«. Mit der persönlich gefärbten Atmosphäre tritt aber keineswegs eine Abkehr von der bisher eingeschlagenen Richtung ein. Die nostalgische Grundstimmung bleibt auch musikalisch erhalten. Ihre Kompositionen wenden die Tradition populärer Songs ins Persönliche; sie holen sozusagen die Bühne ohne Rampenlicht ins Zimmer nebenan.

Mit »Departure Bay« spitzt Krall diesen Ansatz zum Abschluss ihres Albums zu. Unmissverständlich ist hier der private Bezug offen gelegt. Departure Bay heißt eine Bucht vor Kralls Geburtsort Nanaimo auf Vancouver Island vor der Westküste Kanadas. Krall, Jahrgang 1964, thematisiert das Heimkehren, das erneute Aufbrechen und ihre Sehnsucht einst endgültig zurückzukehren. Die nostalgische Stimmung des Textes findet in der Komposition ihre musikalische Entsprechung. Zwar nutzt Krall den herkömmlichen 
Strophe-Refrain-Verlauf, allerdings mit markanten Eingriffen in die erwartete homogene Anlage. Die Harmonik mag dies verdeutlichen: Die Strophe beginnt in f-moll und endet auf Es-Dur im Wechsel mit Des-Dur, womit keineswegs der Refrain vorbereitet, sondern die größtmögliche Distanz zu ihm entwickelt wird. Denn der Refrain beginnt unvermittelt in a-moll. Der Verlust der dauerhaften Heimat ist hier mehr als spürbar: "I just get home, and then I leave again« heißt es vermeintlich lapidar zu Beginn des Refrains. Auch der Refrain an sich besitzt die nostalgische Zerrissenheit. Er mündet nach acht Takten in einen zweiten Teil, der sich in einer erneut anderen Tonart (Es-Dur/c-moll) an den ersten reiht und völlig offen auf c-moll endet. Auch die formalen Proportionen der beiden Refrainteile bilden die zerrissene Offenheit nach. Erstreckt sich der A-Teil über die konventionellen acht Takte, so dehnt Krall den B-Teil auf zehn Takte durch das offene Motiv zu den Worten »Departure Bay« mit dem lang gehaltenen Ton $d^{2}$.

Die nostalgisch verklärte Erinnerung lässt die musikalischen Konturen verschwimmen. Neben der gedehnten Form und der aufgebrochenen Harmonik zeigt auch die Melodik einen immer wieder wechselnden Duktus. Allein im Refrain (B-Teil) folgen auf den eher liedhaften A-Teil gesprochene, fast gemurmelte Motive, die sich bis ins Flehen steigern. Zwar verändert Krall den Refrain bei seiner zweiten und letzten Wiederkehr textlich dahingehend, dass sie eine dauerhafte Rückkehr beschwört: »Just get me there, and one day we'll stay. « Doch die Musik folgt diesem Aspekt nicht.

Letztlich spiegelt sich im nostalgischen Ansatz Kralls auch die medial bedingte Rezeption von Musik mittels Tonträgern wider. Das Musikhören in privater Umgebung wird zu einer besonders persönlichen Erfahrung bei gleichzeitig unendlicher Distanz zur eigentlichen Produktion der Musik an einem unbestimmten oder zumindest entfernten Ort. In der dritten Strophe von »Departure Bay« klingt dies an: »A song plays on the grammophone, and thoughts turn back to life. We took the long way to get back, like driving over Malahat. « Aufgrund der Abgelegenheit ihres Heimatortes Nanaimo auf Vancouver Island war Diana Krall besonders auf Schallplatten angewiesen. Insbesondere die Schallplattensammlung ihres Vaters war dementsprechend prägend für ihre grundlegenden musikalischen Erfahrungen.

\section{Norah Jones}

Nahm Diana Krall erst auf ihrem jüngsten Album eigene Kompositionen in ihr Repertoire auf, setzte Norah Jones gleich mit ihrer ersten CD-Veröffentlichung vornehmlich auf eigene Songs oder auf Stücke, die von Musikern 
ihres unmittelbaren Umfelds stammen, wie Jesse Harris und Lee Alexander. Das überaus erfolgreiche Debut Come Away With Me aus dem Jahr 2002 besaß in vielen Titeln einen Hang zur Country Music. Dass von den wenigen älteren Kompositionen eine auf Hank Williams zurückgeht, ist in diesem Zusammenhang bezeichnend. ${ }^{4}$ Noch erheblich verstärkt hat Norah Jones ihre Hinwendung zur Country Music auf ihrem zwei Jahre später erschienenen Album Feels Like Home. Der programmatische Titel der CD gibt auch musikalisch die Richtung vor. Die Heimat assoziiert Jones mit Musik ländlicher Herkunft; Geborgenheit, Unschuld oder Natürlichkeit werden in ihrer ursprünglichen Einheit als musikalische Ideale entworfen.

Die Kompositionen von Norah Jones oder ihren Bandmitgliedern besitzen keineswegs die Gespanntheit der Songs von Diana Krall und ihrer damit einhergehenden differenzierten Eigentümlichkeiten. Ganz im Gegenteil: Jones' schlichte melodische, harmonische oder formale Ausprägungen (wie auch diejenigen ihrer Mitmusiker) zielen auf eine unmittelbare Wahrnehmung, die fast zum Mitsingen einlädt.

Der Refrain von »Sunrise«, dem Eröffnungsstück des Albums Feels Like Home, gibt dafür in seiner kaum noch zu überbietenden Einfachheit ein prägnantes Beispiel. Norah Jones und Lee Alexander verzichten als Songwriter sogar auf einen Text, gesungen wird allein auf dem Vokal » $\mathrm{u}$ (bzw. »00«). Das in gleichmäßigen Halben mit abschließender Ganzen Note rhythmisierte Viertonmotiv besteht aus den Akkordtönen der Tonika Es-Dur mit dem Einstieg auf der hinzutretenden Sexten: $c^{2}-g^{1}-b^{1}-e s^{1}$. So einfach Rhythmik, Intervallstruktur und auch die homophon gesetzten Akkorde daherkommen, völlig durchsichtig gibt sich die musikalische Idylle jedoch nicht. Das Motiv endet zwar auf es ${ }^{1}$, ist aber harmonisch mit dem As-Dur-Akkord unterlegt und hält die harmonische Anlage ein klein wenig in der Schwebe. Ähnliches geschieht in metrischer Hinsicht durch einen in den durchgehenden 2/2-Takt eingeschobenen 3/2-Takt. Dass das Motiv keine Weiterentwicklung zu einer Melodie erfährt, sondern unverändert dreimal hintereinander erklingt, lässt ihm etwas Fragmentarisches. Durch die nicht gerade zu erwartende Wendung hin zu f-moll in den angehängten Schlusstakten des Refrains wird die nahe gelegte Vertrautheit zusätzlich etwas geöffnet.

Ebenso deutlich wie die Natürlichkeit ist deren Inszenierung; die Idylle wird als schwebende Projektion vorgeführt. Dem entspricht die klangliche Realisierung des Songs mit einem rein akustischen Instrumentarium und vor

4 Auf einen Song von Hank Williams, "I'm So Lonesome I Could Cry«, hat auch Cassandra Wilson auf New Moon Daughter zurückgegriffen. Für Wilson war dies eine Bezugnahme auf ländliche Musik neben anderen; die grundlegende Orientierung am Blues stand deshalb nicht in Frage. 
allem dem Stimmtimbre von Norah Jones. Ihr Gesang mit gehauchter Kopfstimme und entsprechender Laid-back-Phrasierung changiert zwischen unbeschwerter Gelassenheit und fragilem Selbstbewusstsein. Der Song "Sunrise « und seine Interpretation lassen ein musikalisches Kleinod entstehen, eine kunstvoll gestaltete, zierliche Kleinigkeit, eine Kostbarkeit.

Vergleichbares trifft auch auf die übrigen Titel des Albums zu, selbst auf diejenigen, die nicht aus der Feder von Jones oder ihren Mitmusikern stammen. Die Wahl des ebenfalls äußerst schlicht gehaltenen Songs »Long Way Home « von Kathleen Brennan und Tom Waits, 2002 für den Film Big Bad Love geschrieben, erweist sich als ebenso überzeugend. Der Weg nach Hause dauert hier nicht nur lang, sondern unendlich lang. Die Musik schlendert dem Text folgend viele Takte auf der Tonika vor sich hin: »| stumble in the darkness. « Mit dem Wechsel hin zur Moll-Parallele auf fis zu den Worten »to show the way back home « macht Waits deutlich, dass die Heimat mehr eine sentimentale Projektion als ein erreichbares Ziel ist. ${ }^{5}$

Sowohl die Interpretation von Waits als auch diejenige von Jones bestätigen klanglich die Schlichtheit des Songs. Durch Waits' Gesang mit seiner extrem rauen, gebrochenen Stimme erhält das musikalische Schlendern sein eher zielloses Landstreicher-Flair. Bei Jones wandelt sich der gehauchte, vergeblich anmutende Sprechgesang zur optimistischer klingenden Kopfstimme. Denn das Umherstreifen hat auch seine positive Seite, wie in der letzten Strophe aufgezeigt wird: wenn man es nämlich zu zweit unternimmt. Jones unterstreicht diese Hoffnung, indem sie ihrem Gesang zunächst gelegentlich und schließlich in der letzten Strophe durchgehend eine zweite Gesangsstimme hinzufügt.

Entsprechend der Musik zeigt das Starimage Norah Jones als natürlich, spontan, unprätentiös. Bezeichnend sind hier die Musikvideos zu den Songs »What Am I To You?« und »Those Sweet Words«. In erstgenanntem sind vor allem gefilmte Schnappschüsse jenseits des Konzertpodiums eingestreut, die Jones scheinbar als lebenslustigen, gelegentlich zu Späßen aufgelegten Menschen im Umgang mit ihren Mitmusikern vorführen. Im Clip zu »What Am I To You?« spielt Jones eine schüchterne Karaoke-Sängerin in einem kleinen Club. Die Inszenierungen setzen auf Natürlichkeit und Unschuld, sind darum aber nicht minder künstlich.

5 Um genau zu sein: Die Einleitung präsentiert die Begleitung in A-Dur, die dann harmonisch unverändert die ersten sieben Takte beibehalten wird. Waits bereitet den Wechsel hin zum fis-moll-Akkord dann im achten Takt vor. Jones hält sogar noch im achten Takt am Grundton $a$ fest und wechselt direkt nach fis-moll im neunten Takt. 
Auf dem ersten Studioalbum von Norah Jones wirkte Gitarrist Bill Frisell beim Song »The Long Day Is Over« mit. Ein bezeichnendes Detail, denn Frisell, einst als Mitglied der New Yorker Downtown Avantgarde um John Zorn bekannt geworden, hatte seit etwa Mitte der 1990er Jahre eine nicht minder sentimentale Ausrichtung hin zur Vergangenheit praktiziert. Mit seinen 1995 auf CD veröffentlichten Begleitmusiken zu den Buster-Keaton-Filmen The High Sign, One Week und Go West bevorzugte Frisell hier die Stummfilmära in ihrer melancholisch-humorvollen Ausprägung. Musikalisch noch weit deutlicher gerät Frisells Nostalgie mit seiner Hinwendung zur Country Music, wie er sie 1997 programmatisch auf seinem Album Nashville vorstellte und seitdem in verschiedensten Ausprägungen fortsetzte, zuletzt 2005 sogar als Begleiter des Country-Duos Chip Taylor und Carrie Rodriguez.

Die Nostalgie im Jazz ist offenbar nicht auf einige wenige Beispiele beschränkt, sondern entpuppt sich als einer der wesentlichen Trends zumindest der vergangenen fünfzehn Jahre. Dass damit nicht einfach retrospektiv ausgerichtete jazzmusikalische Phänomene gemeint sind, sollte mit der Besprechung der Musik von Cassandra Wilson, Diana Krall und Norah Jones konkretisiert werden. Das New Orleans-Revival der 1940er Jahre oder das Bebop-Revival der 1980er Jahre beispielsweise waren nicht per se nostalgisch, nur weil hier die Musik früherer Zeiten aufgegriffen wurde. Bunk Johnson oder Louis Armstrong mit seinen All Stars oder Jahrzehnte später Wynton Marsalis als die prominentesten Protagonisten der genannten Revival-Bewegungen waren alles andere als melancholisch aufgelegt. Die Achtung vor der musikalischen Vergangenheit und das Bemühen um deren lebendige Bewahrung gingen hier nicht mit einer sentimentalen Sehnsucht nach den "guten, alten Zeiten« einher. Auch wenn in einigen Texten durchaus nostalgische Themen auftauchen, so lässt sich noch längst nicht von musikalischer Nostalgie sprechen wie im Fall von Wilson, Krall und Jones. Deren entsprechende kreative Projektionen besitzen eine jeweils ganz eigene Qualität, die sich in ihren Images überspitzt bündelt, sei es die natürliche Leichtigkeit von Norah Jones, die plakative Eleganz von Diana Krall (inzwischen mit einem Hang ins Persönliche) oder die bodenständige, ursprüngliche Sinnlichkeit von Cassandra Wilson.

Viel war bisher die Rede von verschiedenen Genres populärer Musik, vom Blues über Bossa Nova bis zur Country Music, auf die die erwähnten Jazzmusikerinnen und -musiker zurückgegriffen haben. Inwiefern ist dann in diesem Zusammenhang die Bezeichnung »Jazz« eigentlich überhaupt noch 
sinnvoll? Oder besser gefragt: Was ist dann mit »Jazz « gemeint? Bill Frisell gibt hierauf eine interessante Antwort:

»For me jazz is more a process. It's the way you go at the music, rather than a style and it seems these days, it's become more defined up to just music in general, rather than just zeroing in on the one instrument« (Frisell, zit. n. Jung 2004).

"Jazz« wäre dann nicht mehr als spezifisches Genre mit verschiedenen stilistischen Materialausprägungen zu begreifen, sondern als besondere Art des Umgangs mit bereits bestehenden Stilen und musikalischen Materialien. Damit läge zumindest ein konsequentes Weiterdenken der uferlosen Ausdifferenzierung dessen vor, was einst als »Jazz« bezeichnet wurde.

Aber vielleicht ergibt sich aus Entwicklungen im Jazz der letzten Jahre bzw. Jahrzehnte sogar ein grundlegend veränderter Blick auf die Jazzgeschichte insgesamt. Hatte nicht schon Jelly Roll Morton, der sich selbst gern als »Erfinder des Jazz « bezeichnete, in seinen Erinnerungen an das New Orleans Anfang des 20. Jahrhunderts geschrieben:

»In der Jazzmusik findet man die besten musikalischen Ideen aus den bedeutendsten Opern, Symphonien und Ouvertüren wieder. Das gehört zum Schönsten im Jazz. [...] Ich verarbeitete eine ganze Reihe derartiger Stücke zu Jazznummern, wobei ich verschiedene kleine Variationen und Ideen zur Verkleidung der Melodien verwendete. Ich habe zum Beispiel den Tiger Rag aus einer alten Quadrille geschöpft, welche ursprünglich in verschiedenen Tempi gesetzt war« (Morton/Lomax 1992: 71f.).

Die musikalische Nostalgie - zumindest dies lässt sich festhalten - ist als Genres-, Generationen- und auch Gender-übergreifender Trend mitbestimmend für den Jazz der letzten Jahre. Die sentimentale Sehnsucht nach vergangenen Orten und Zeiten kommt musikalisch als pointiert stilisierter Nachhall daher.

\section{Literatur}

Haden, Charlie (1992). Begleittext zur CD von Charlie Haden Quartet West: Haunted Heart. Verve 513 078-2.

Jung, Fred (2004). »A Fireside Chat with Bill Frisell.«In: www.allaboutjazz.com/ php/article_print.php?id=1156 (Zugriff: 22.8.2005).

Kampmann, Wolf (2000). "Craig Street. Produktivkräfte der Musikwelt (Folge 1).« In: Jazzthing, Nr. 32 (Februar/März), S. 54-56.

Morton, Jelly Roll / Lomax, Alan (1992). Doctor Jazz. Eine Autobiographie. Übersetzt von Fritz Herdi. Hamburg: Luchterhand.

Reid, Jamie (2002). Diana Krall. The Language of Love. Markham: Quarry. 
Simanga, Michael (2002). Begleittext zu Cassandra Wilsons CD Belly Of The Sun. Blue Note 724353507220 .

\section{Diskographie}

Astaire, Fred (2003). Top Hat, White Tie \& Tails. Complete Recordings, Vol. 3, 1933-1936. Naxos, 8.120718.

Charlie Haden Quartet West (1992). Haunted Heart. Verve,513 078-2.

Charlie Haden Quartet West (1994). Always Say Goodbye. Verve, 521 501-2.

Frisell, Bill (1995). Music For The Films Of Buster Keaton. Go West. Elektra Nonesuch, 7559 79350-2.

Frisell, Bill (1995). Music For The Films Of Buster Keaton. The High Sign. One Week. Elektra Nonesuch, 7559 79351-2.

Frisell, Bill (1997). Nashville. Nonesuch, 7559-79415-2.

Jones, Norah (2002). Come Away With Me. Blue Note, 724353208820.

Jones, Norah (2004). Feels Like Home. Blue Note, 724386652225.

Krall, Diana (1993). Stepping Out. The Early Recordings. Justin Time/GRP/ Universal 059 825-2.

Krall, Diana (1995). Only Trust Your Heart. GRP/Universal 059 810-2.

Krall, Diana (1996). All for You. A Dedication To The Nat King Cole Trio. GRP/ Impulse, IMP 11642.

Krall, Diana (1997). Love Scenes. Impulse, IMP 12342.

Krall, Diana (1999). When I Look In Your Eyes. Verve, 050 304-2.

Krall, Diana (2001). The Look Of Love. Verve, 549 846-2.

Krall, Diana (2004). The Girl In The Other Room. Verve, 0602498622469.

Sinatra, Frank (1961). Ring-A-Ding Ding! Reprise FS 1001.

Sinatra, Frank (1967). Francis Albert Sinatra \& Antonio Carlos Jobim. Reprise, FS 1021.

Taylor, Chip / Rodriguez, Carrie (2005). Red Dog Tracks. Train Wreck, TW 022.

Waits, Tom (2001). »Long Way Home«. Auf: Big Bad Love. Music From The Motion Picture Soundtrack. Nonesuch, 7559-79637-2.

Wilson, Cassandra (1987/2002). Days Aweigh. JMT/Winter \& Winter 910 012-2.

Wilson, Cassandra (1988). Blue Skies. JMT/Polydor 834419-2.

Wilson, Cassandra (1992). After The Beginning Again. JMT/Polydor, 514 001-2.

Wilson, Cassandra (1992). Dance To The Drums Again. Rebel/DIW/Columbia, COL 472972.

Wilson, Cassandra (1993). Blue Light 'Til Dawn. Blue Note, CDP 077778135722.

Wilson, Cassandra (1994). New Moon Daughter. Blue Note, CDP 724383718320.

Wilson, Cassandra / Terrasson, Jacky (1997). Rendezvous. Blue Note, 7243855484 20.

Wilson, Cassandra (1999). Travelling Miles. Blue Note, 724385412325.

Wilson, Cassandra (2002). Belly Of The Sun. Blue Note, 724353507220.

Wilson, Cassandra (2003). Glamoured. Blue Note, 724359095127. 


\title{
Songbooks
}

Jones, Norah (2004). Feels Like Home. Milwaukee: Hal Leonard.

Krall, Diana (2003). The Collection. London: Wise.

Krall, Diana (2004). The Girl In The Other Room. Milwaukee: Hal Leonard.

\begin{abstract}
Nostalgic themes presented musically by combining old styles are a recent trend in jazz. Among others, Cassandra Wilson, Diana Krall and Norah Jones are the leading musicians in this context. Although nostalgia has been treated in different musical genres for a long time before, there has not been a musical treatment concerning the styles of former decades. Now, jazz musicians create certain nostalgic aspects musically: They do not revive the former styles as in revival movements, but they create sound echoes, making audible their yearning for but also their distance to the "good old days «.
\end{abstract}

